\title{
Tailored Sheared Blanks Produced by Incremental ECAP
}

\author{
Andrzej Rosochowski ${ }^{1, a^{*}}$, Lech Olejnik ${ }^{2, b}$, Malgorzata Rosochowska ${ }^{3, c}$ \\ ${ }^{1}$ Design, Manufacture and Engineering Management, University of Strathclyde, James Weir \\ Building, 75 Montrose Street, Glasgow G1 1XJ, United Kingdom \\ ${ }^{2}$ Institute of Manufacturing Processes, Warsaw University of Technology, ul. Narbutta 85, 02-524 \\ Warsaw, Poland \\ ${ }^{3}$ Advanced Forming Research Centre, University of Strathclyde, 85 Inchinnan Drive, Inchinnan, \\ Renfrewshire, PA4 9LJ, United Kingdom \\ aa.rosochowski@strath.ac.uk, 'l.olejnik@wip.pw.edu.pl, 'm.rosochowska@strath.ac.uk
}

Keywords: Severe plastic deformation, incremental forming, equal channel angular pressing, tailored blanks.

\begin{abstract}
Incremental equal channel angular pressing (I-ECAP) is a process used for production of continuous ultrafine grained bars, plates and sheets. Normally the thickness of the processed billet is kept unchanged in consecutive passes to enable repetitive insertion into the same die. This is achieved by controlling the bottom dead centre of the reciprocating punch. However, if a final product requires being thinner and therefore longer, the bottom position of the punch can be lowered before the last pass. Going further, the bottom position of the punch can be changed during the process, which opens up a possibility to vary billet thickness along its length. Such a product, especially sheet, can serve as a preform for further metal forming operations and is known as tailored blank. This paper will show examples of varying thickness sheets produced by different configurations of I-ECAP. Experimental and finite element results will be presented.
\end{abstract}

\section{Introduction}

Bulk metals with ultrafine grained (UFG) structure, characterised by the average grain size $<1$ $\mu \mathrm{m}$, draw substantial attention due to their unique mechanical and physical properties. A popular method of producing bulk UFG metals is severe plastic deformation (SPD) [1]. In this method, a very large plastic deformation (true strain $>3$, depending on the metal) "subdivides" coarse metal grains into sub-micrometre size grains. Unlike traditional metal forming processes, SPD processes retain the shape of the billet.

Equal channel angular pressing (ECAP), originally proposed by Segal et al. under the name of equal channel angular extrusion [2], is the most popular SPD process used to produce UFG metals. In this process, a square or cylindrical billet is pushed by a punch through an input constant profile channel to an output channel of the same profile orientated at an angle $\geq 90^{\circ}$ to the input channel. Plastic deformation of the material is caused by simple shear in a thin layer along the diagonal plane at the channel crossing. The process is usually repeated several times with the billet being rotated about its axis between consecutive passes. The process is simple in terms of tooling and machines used, however, it suffers from poor material utilisation because undeformed material at billet ends makes a large proportion of a typical short billet.

Incremental ECAP (I-ECAP) was introduced in 2005 and subsequently patented [3,4]. The process was first revealed publically in 2007 [5]. It is based on separating material feeding and deformation in classical ECAP by using a reciprocating punch and an incremental, synchronised feeder. Such tool kinematics substantially reduces the feeding force, which in turn, enables processing continues long billets. The process was proved to work for square cross-section bars [6], plates $[7,8]$ and sheets $[9,10]$. It was also considered for processing tubes [11], reducing friction by working with converging billets [12] and splitting a rectangular cross-section billet [13]. 
UFG billets usually require further forming into useful preforms and products, for example, by rolling, extrusion, drawing or other metal forming processes. These additional forming operations do not only change the shape of billets but also further improve their mechanical properties above the saturation limit often characterising repetitive severe plastic deformation [14]. A special class of products are tailored blanks often used in the automotive industry [15]. They are sheet blanks with either local variation of thickness or properties. The most popular approach is based on differentiation of sheet thickness in otherwise uniform material. This can be achieved by butt welding of sheets with different thickness or lap welding to create patches to create so-called tailored welded blanks (TWB). The welding technology is well established but has its limitations such as introducing a discontinuity causing problems during sheet metal forming and product exploitation. Also, some metals are not easily weldable. An alternative approach is based on flexible rolling, where a roll gap changes programmatically during the process, to produce tailored rolled blanks (TRB) [16]. This approach usually leads to a long thickness transition zone, as the recommended thickness change slope is 1:100. The current paper investigates feasibility of yet another approach, based on I-ECAP with changing sheet thickness, to produce tailored sheared blanks (TSB).

\section{Incremental ECAP of sheets}

In this paper, two cases of processing sheets with I-ECAP are considered. The first case, described in [9], involves two metal strips with $2 \times 50 \mathrm{~mm}$ cross-section separated with a segmented feeder (Fig. 1). The feeder serves three functions. Its bottom segment has a step on each side, which enables feeding the strips upwards and prevents their backward movement. The segments also play the role of a movable die, which travels together with the strips and, therefore, does not introduce friction. Finally, they also guide the punch by entering a gap in the punch. Since the gap has a limited length, the top segment has to be pushed out to the side as soon as it appears above the die. Each face of the punch has a half-spike on its edge (too small to be seen in Fig. 1), to improve the material flow. The die is also divided into segments, which are kept together by the dowel pins and bolts. Two of these segments are taller to provide side walls for the horizontal channel to secure plain strain condition and guide the punch.

(a)

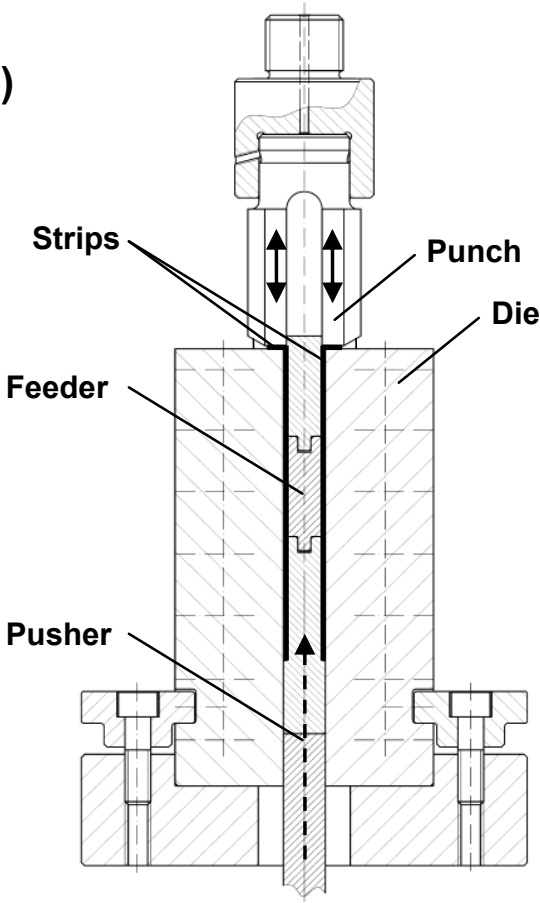

(b)

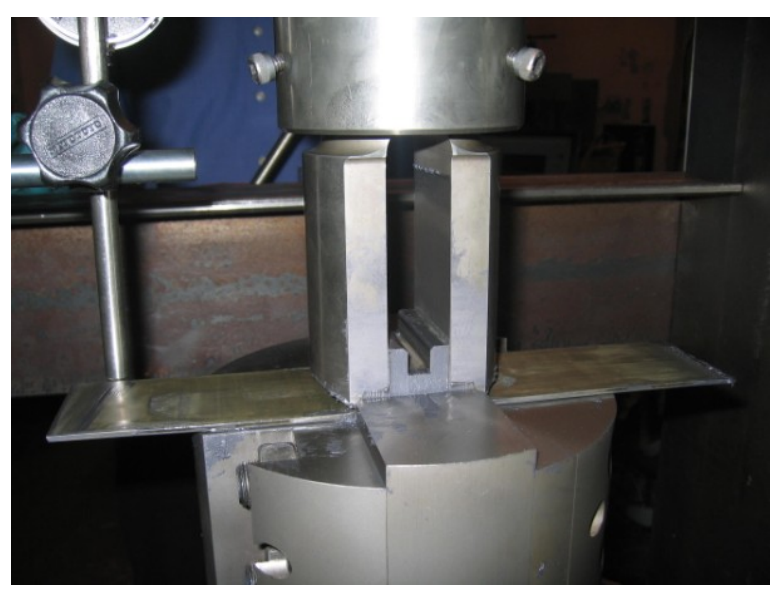

(c)

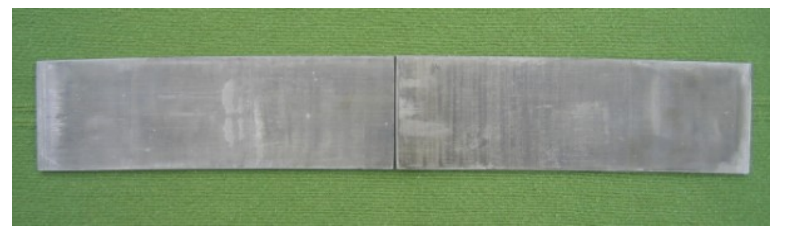

Fig. 1: I-ECAP of two sheets; tool design (a), final stage of process (b) and processed sheets (c). 
The second case of using I-ECAP for sheets involves a single sheet/plate with a $3 \times 62 \mathrm{~mm}$ crosssection [10]. As shown in Fig. 2, a sheet strip is fed incrementally by a pusher and deformed by a reciprocating punch, which moves at an angle of $30^{\circ}$ to the feeding direction. The input channel is defined by a stationary die and a clamp. At each cycle, the sheet is fed by a small distance, then stopped and deformed by simple shear with the punch. After several cycles, the whole length of the sheet is processed so it can be removed from the output channel.

(a)

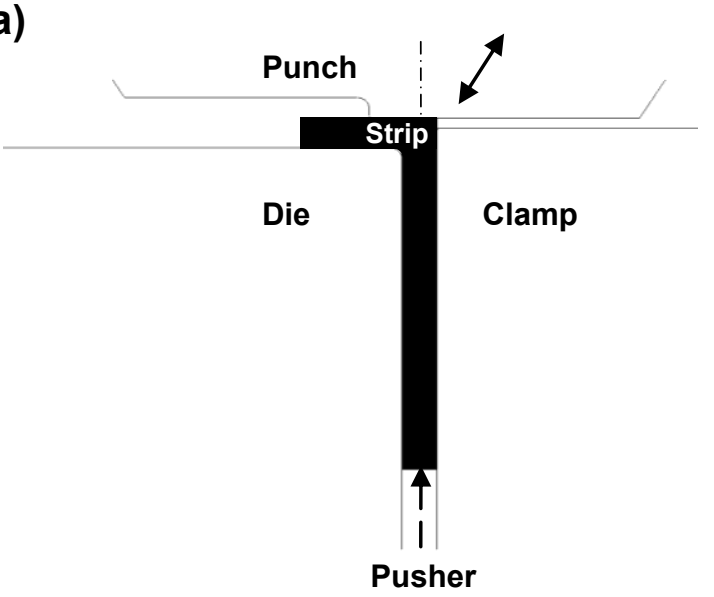

(b)

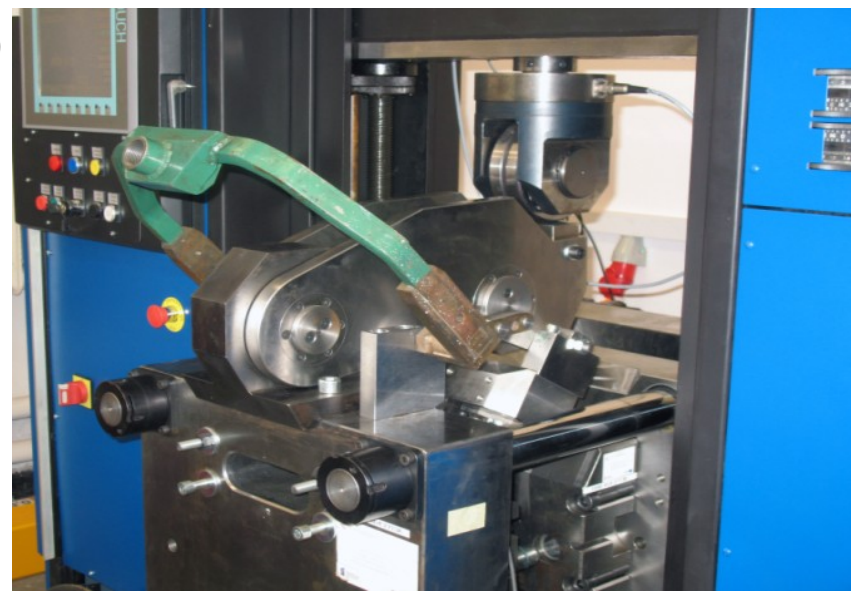

Fig. 2: Tool configuration (a) and photograph (b) of I-ECAP machine for a single sheet/plate.

\section{Producing sheets with variable thickness}

Following the concept of severe plastic deformation, which does not change the shape of a billet processed, only constant thickness sheets have been produced by I-ECAP [9,10]. Since sheet thickness depends on the bottom dead centre of the reciprocating punch, it has to be kept constant so the initial sheet thickness is maintained throughout the process. However, it can also be lowered before the last pass to get sheet thickness smaller than the initial one but still constant. Another option is to change sheet thickness during the process by altering the bottom dead centre of the punch gradually or stepwise. To check feasibility of this new approach, a preliminary trial with 190mm long Al 1050 sheet has been performed using I-ECAP configuration in Fig.1; the cycle frequency was $1 \mathrm{~Hz}$. The bottom dead centre of the punch has been changed manually during the process in order to reduce sheet thickness in several steps, from $2.20 \mathrm{~mm}$ (nominal sheet thickness was $2 \mathrm{~mm}$ ) down to $0.60 \mathrm{~mm}$. As shown in Fig. 3, the results are encouraging despite some bending of the sheet taking place at the moments of thickness change. The process can be used just once to produce a variable thickness sheet/strip with deformed material structure or be the last one in a series of constant thickness I-ECAP passes used to refine grain structure.

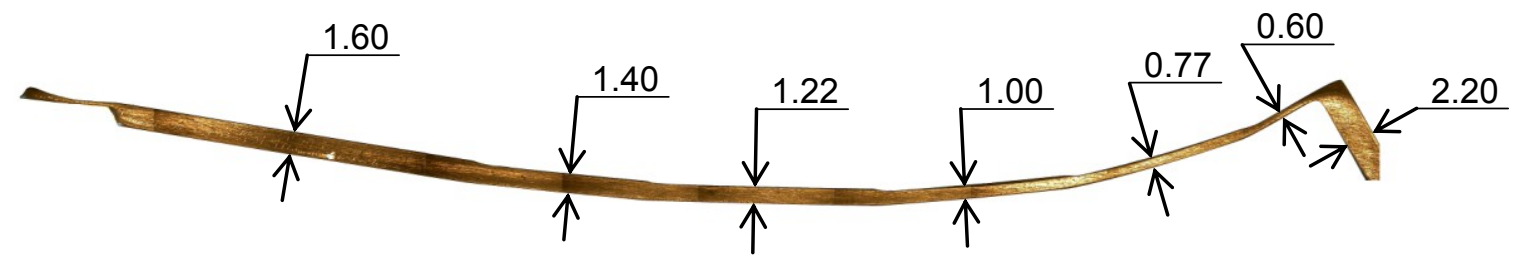

Fig. 3: I-ECAP (as in Fig. 1) carried out to change thickness of aluminium 
More comprehensive trials have been performed using I-ECAP of a single sheet/plate as presented in Fig. 2. The material was the same Al 1050 but thicker (initial thickness $3 \mathrm{~mm}$ ) and shorter (initial length $105 \mathrm{~mm}$ ), which reduced the tendency to bending. The lowest punch position was controlled programmatically to realise a given sequence of sheet thickness changes; the cycle frequency was $0.1 \mathrm{~Hz}$. Fig. 4 presents some of the possible profiles and the forming forces recorded. Two cases were tested, one with the increasing sheet thickness (Fig. 4a), producing steps on the die side, and another one with initially decreasing and then increasing sheet thickness (Fig. $4 \mathrm{~b})$ leading to steps produced on the punch side and later on the die side. The force profiles followed those changes, with higher forces recorded for larger thickness reduction.
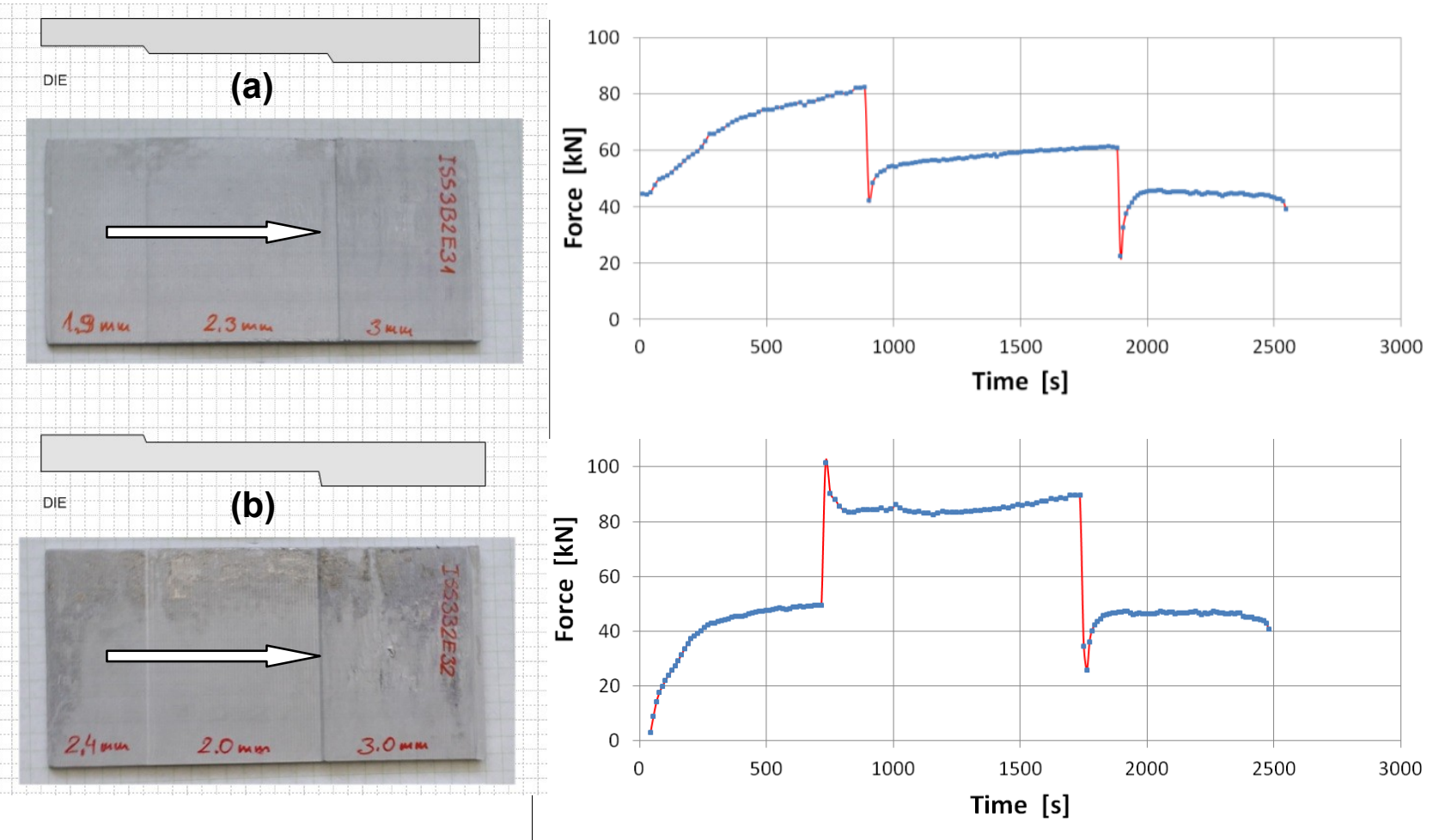

Fig. 4: Three-step tailored blanks produced by I-ECAP and the forces recorded.

\section{Finite element simulation}

To get a better insight into the process, finite element (FE) simulation has been carried out for a process similar to that illustrated in Fig. 2 but optimised for the uniform material flow. The material modelled in Abaqus/Explicit was Al 1050. It was modelled as an elastic-plastic, isotropic, Huber/Mises material. Friction was assumed to follow Coulomb's law with friction coefficient $\mu=0.1$. The punch was reciprocating at $45^{\circ}$ to the feeding direction, with a high frequency (to shorten calculation time) and double amplitude of $2 \mathrm{~mm}$. The feeding stroke was $0.6 \mathrm{~mm}$. The sequence of changing sheet sickness was $2.4 \mathrm{~mm} \rightarrow 2.0 \mathrm{~mm} \rightarrow 3 \mathrm{~mm}$ as in Fig. 4b. Fig. 5a presents equivalent strain distribution obtained in this simulation while Fig. $5 \mathrm{~b}$ enables comparison of the calculated profile with the real one (magnified in the normal direction for better visualisation). The profiles look similar despite a shorter sheet used for FE simulation to save the computation time. The sheet steps are created on the punch side when the thickness decreases and on the die side when the thickness increases. 
(a)

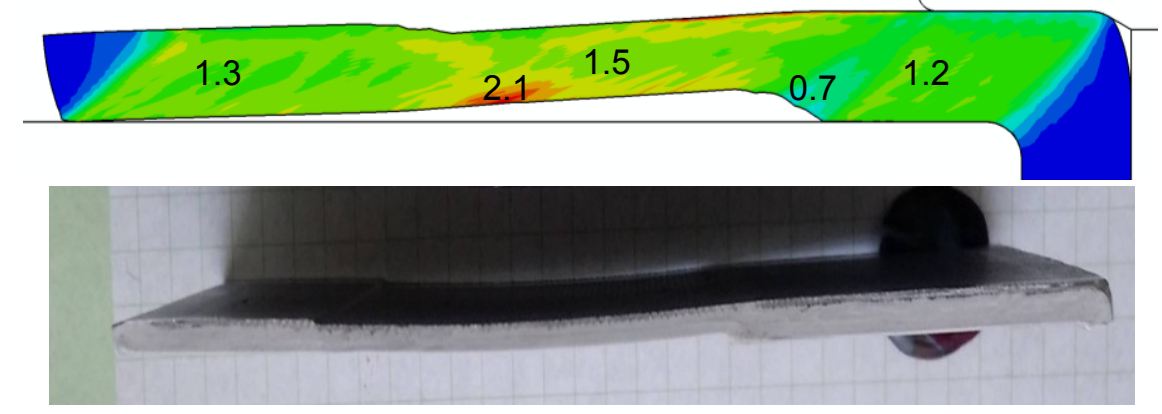

Fig. 5: FE simulation of I-ECAP of variable thickness sheet (a) and photo of real sheet (b).

\section{Discussion and conclusions}

I-ECAP proved its suitability for producing constant thickness sheets, having the same dimensions as the initial sheets, in the past. However, taking into account kinematics of the I-ECAP tools, there is no problem with producing sheets with constant but reduced thickness in the last pass of the process. The research reported here goes further and investigates a possibility of producing sheets with varying thickness, which can be referred to as tailored sheared blanks (TSB). Two IECAP configurations have been tested to obtain TSBs.

The first one, shown in Fig. 1, proved feasibility of manually changing the lowest punch position during the process to reduce sheet thickness by steps, from the initial value of $2.2 \mathrm{~mm}$ to the final value of $0.6 \mathrm{~mm}$ (Fig. 3). The only adverse effect observed was bending of the sheet towards the punch. This would require straightening if flat blanks were to be used for further processing. The following experiment, shown in Fig. 2, used a different tool configuration, with a single sheet and a punch reciprocating at an angle of $30^{\circ}$ to the feeding direction. This time, the lowest punch position was controlled programmatically to get various thickness profiles. It appeared that the sheet steps were created on either punch or die side depending on whether thickness was decreased or increased (Fig. 4).

The process modelled by FE simulation was slightly different from that in Fig, 2 in order to facilitate material flow at the punch/clamp interface and avoid numerical problems. This has been achieved by increasing the punch angle to $45^{\circ}$ and letting clamp to follow punch to avoid a gap between these tools. Due to friction, the upward movement of clamp lifted sheet towards punch during the feeding stage. Also, the changing thickness was realised by moving die as an alternative to moving punch. However, those differences did not change the fundamental pattern of material flow. The thickness changes observed were like in the experiment (Fig.5). An additional insight to the process was provided by distribution of equivalent strain, which changed from approximately 1.3 through 1.5 to 1.2 (Fig. 5). Since equivalent strain in I-ECAP with changing sheet thickness results from simple shear combined with plain strain compression, those values should be more like 1.4, 1.6 and 1.15. The lower calculated values were due to the excessive feeding stroke of $0.6 \mathrm{~mm}$, which constituted $30 \%$ of the lowest sheet thickness of $2 \mathrm{~mm}$. This led to non-uniform equivalent strain distribution along the sheet and lowering the average value of this strain. Another nonuniformity was observed on the die side of sheet in the form of increased strain at the thickness reduction step and decreased strain at the thickness increment step.

Comparing TSBs with TRBs (or tailored hammered blanks), there is a scope for much larger strain to be induced since, in addition to usually small thickness strain, simple shear contributes with equivalent strain of 1.15 ; this can produce much stronger material and potentially a refined grain structure. Another advantage over TRBs is a controllable thickness transition zone which, if necessary, can be very short to enable frequent thickness changes. 


\section{References}

[1] A. Azushima, R. Kopp, A. Korhonen, D.Y. Yang, F. Micari, G.D. Lahoti, P. Groche, J. Yanagimoto, N. Tsuji, A. Rosochowski, A. Yanagida, CIRP Annals - Manufacturing Technology 57 (2008) 716-735.

[2] V.M. Segal, V.A. Reznikov, A.E. Drobyshevskiy, V.I. Kopylov, Russian Metallurgy 1 (1981) 99-105.

[3] A. Rosochowski, European Patent 1861211 (2012)

[4] A. Rosochowski, U.S. Patent 8,631,673 (2014)

[5] A. Rosochowski, L. Olejnik, FEM simulation of incremental shear, in: E. Cueto, F. Chinesta (Eds), Proc. of the 10th International Conference on Material Forming, Esaform 2007, April 18-20, 2007, Zaragoza, Spain, AIP Proceedings 907, 2007, p. 653-658.

[6] A. Rosochowski, L. Olejnik, M. Richert, Double-billet Incremental ECAP, Materials Science Forum 584-586 (2008) 139-144.

[7] A. Rosochowski, L. Olejnik, M. Richert, Incremental ECAP of plates, Materials Science Forum 584-586 (2008) 108-113.

[8] A. Rosochowski, L. Olejnik, Incremental ECAP of thick continuous plates - machine and initial experiments, 6th International Conference on Nanomaterials by Severe Plastic Deformation, NanoSPD6, 30 June - 4 July 2014, Metz, France, IOP Conf. Series: Materials Science and Engineering 63 (2014) 012010.

[9] A. Rosochowski, M. Rosochowska, L. Olejnik, B. Verlinden, Incremental equal channel angular pressing of sheets, Steel Research International 81/9 (2010) 470-473.

[10]L. Olejnik, W. Chromiński, A. Rosochowski, M. Lipińska, M. Lewandowska, Incremental ECAP as a novel tool for producing ultrafine grained aluminium plates, 6th International Conference on Nanomaterials by Severe Plastic Deformation, NanoSPD6, 30 June - 4 July 2014, Metz, France, IOP Conf. Series: Materials Science and Engineering 63 (2014) 012004.

[11]A. Rosochowski, L. Olejnik, Incremental ECAP of tubular components - FE simulation, Proc. of the $14^{\text {th }}$ Esaform Conference on Material Forming, Belfast, United Kingdom, 27-29 April 2011, edited by G. Menary, AIP Proceedings 1353, pp. 517-522

[12]A. Rosochowski, L. Olejnik, Incremental ECAP with converging billets, Key Engineering Materials 554-557 (2013) 869-875.

[13]A. Rosochowski, M. Rosochowska, L. Olejnik, Severe plastic deformation by incremental angular splitting, Journal of Materials Science 48/13 (2013) 4557-4562.

[14]L. Olejnik, M. Kulczyk, W. Pachla, A. Rosochowski, Hydrostatic extrusion of UFG aluminium, International Journal of Material Forming 2/Suppl. 1 (2009) 621-624.

[15]M. Merklein, M. Johannes, M. Lechner, A. Kuppert, A review on tailored blanks - Production, applications and evaluation, Journal of Materials Processing Technology 214 (2014) 151-164.

[16]R. Kopp, C. Wiedner, A. Meyer, Flexibly rolled sheet metal and its use in sheet metal forming, Advanced Materials Research 6-8 (2005) 81-92. 